\title{
COVID-19 as the trigger for respiratory failure in neuromuscular diseases
}

\author{
COVID-19 como gatilho para falência respiratória em pacientes com doenças \\ neuromusculares
}

\author{
Marta Rodrigues de CARVALHO', Beatriz Schmidt DAL BERTO', Priscila Leite SANTOS', \\ Guilherme Coelho de AZEVEDO', Rubens Nelson Morato FERNANDEZ1', Talyta GRIPPE',2
}

Dear Editors,

We read with great interest the article by Studart-Neto et al. ${ }^{1}$, which presented the importance of recognizing neurological conditions that can worsen the prognosis of SARS-CoV-2 infection. We acknowledge the notable work demonstrating that knowledge in neurology is relevant for better treatment and follow-up of the disease.

We aim to reveal the importance of early recognition of symptoms and signs of neuromuscular disorders (NMD) in young COVID-19 patients, in whom the disease has a greater possibility of progressing into respiratory failure. Since it is not an expected outcome in this age group, we present two cases in which COVID-19 triggered disease decompensation and we suggest considering NMD in diagnosing young patients with unexpected respiratory failure due to SARSCoV-2 infection.

The first case is a 23-year-old man who complained of weakness, associated with fatigue, muscle pain, and history of falls. Weakness was first attributed to obesity. In 7 months, he lost $40 \mathrm{~kg}$ with restrictive diet; with no improvement of his weakness. Electromyography (EMG) suggested myopathy as the primary diagnosis. He denied ocular symptoms so far and was due for a muscle biopsy when he experienced marked worsening of weakness, dysphagia, and dysphonia. The rt-PCR of nasopharyngeal secretion returned positive for SARS-CoV-2; he was then transferred to our unit. Upon admission, mild ptosis was observed (Figure 1). He underwent orotracheal intubation due to hypercapnic respiratory failure.

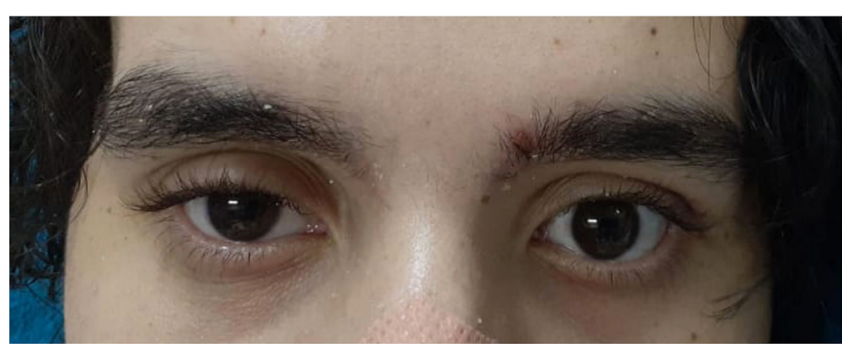

Figure 1. Patient 1 face — right before the intubation, with a mild ptosis.

The patient had normal creatine phosphokinase (CPK) levels. Mild improvement was observed after the intramuscular neostigmine test. Another EMG examination was performed, revealing a decrement greater than $10 \%$ at $3-\mathrm{Hz}$ repetitive stimulation in the ulnar nerve (Figure 2) and no evidence of myopathic motor unit potentials, although this can be a feature encountered in EMG of myasthenic patients. The anti-acetylcholine receptor antibody was positive. Pyridostigmine therapy was then started. He received 6-mg dexamethasone for 5 days to treat COVID-19. His complications included pneumonia, and treatment involved the use of meropenem, polymyxin, daptomycin, vancomycin, ertapenem, tigecycline, linezolid, tazocin, ceftriaxone, and amikacin, which can be considered triggers for myasthenic crisis. Ventilatory status improvement was noted; however, a tracheostomy was required. After 36 days, he was transferred to the neurology ward. Dysphagia, dyspnea, and

\footnotetext{
${ }^{1}$ Hospital de Base do Distrito Federal, Neurology and Neurophysiology Unit, Brasília DF, Brazil.

${ }^{2}$ Centro Universitário de Brasília, Faculty of Medicine, Brasília DF, Brazil.

Marta Rodrigues de CARVALHO (iD https://orcid.org/0000-0002-8616-6735; Beatriz Schmidt DAL BERTO (iD) https://orcid.org/0000-0002-5789-5322;

Priscila Leite SANTOS ID https://orcid.org/0000-0003-1251-401X; Guilherme Coelho de AZEVEDO (D) https://orcid.org/0000-0001-6902-4199;

Rubens Nelson Morato FERNANDEZ (iD https://orcid.org/0000-0003-2978-0269;Talyta GRIPPE (iD https://orcid.org/0000-0003-3126-8002

Correspondence: Talyta Grippe; E-mail: talytagrippe@gmail.com

Conflict of interest: There is no conflict of interest to declare.

Authors' contributions: TG: conceived the presented idea. MRC, GCA, PLS, and BDSCB: collected the data. MRC: wrote the first draft. All authors discussed the results and significantly contributed to the final manuscript. TG and RNMF: made the final revision.

Received on September 30, 2020; Received in its final form on November 17, 2020; Accepted on November 23, 2020.
} 


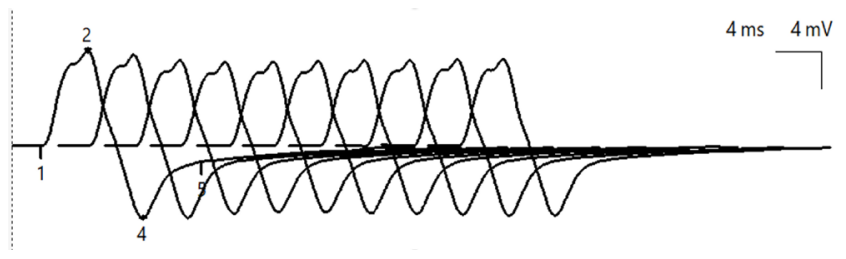

Figure 2. Repetitive stimulation with 11\% decrement between 1 and 4 motor potentials; ulnar nerve — right side.

generalized fatigue were further noted; hence, intravenous immunoglobulin (IVIg) was administered and azathioprine started. Significant clinical improvement was observed 2 weeks later.

The second case is a 14-year-old girl who has been followed by psychiatry clinics for initial symptoms of depression for 3 months, evolving with hallucination, loss of self-care and appetite within the last month. She was under treatment with sertraline and risperidone when she developed generalized weakness and apathy. Following a diarrheic episode for 1 week, her laboratory tests showed high glutamic oxaloacetic transaminase and positive IgG serology for SARS-CoV-2. She received no specific treatment for SARS-CoV-2 infection. Previous drugs were replaced by lithium and mirtazapine, with no improvement in symptoms. She was then admitted to the psychiatry unit.

A neurological consultation was requested due to a $\mathrm{CPK}$ of 5000. On the first physical examination, she was apathetic and had proximal weakness with hyporeflexia and no sensory symptoms. Her consciousness rapidly deteriorated. Blood gas analysis showed PCO2>100; she underwent an orotracheal intubation. EMG showed a myopathic pattern (Figure 3), without significant decrement during the $3-\mathrm{Hz}$ stimulation test. Lithemia was within normal values (0.66 mmol/L). Her rheumatologic panel showed positive antinuclear antibodies (ANA), SSB, SSA, and Antineutrophil cytoplasmic antibodies (P-ANCA) antibodies and low C3 complement levels. She was diagnosed with systemic lupus

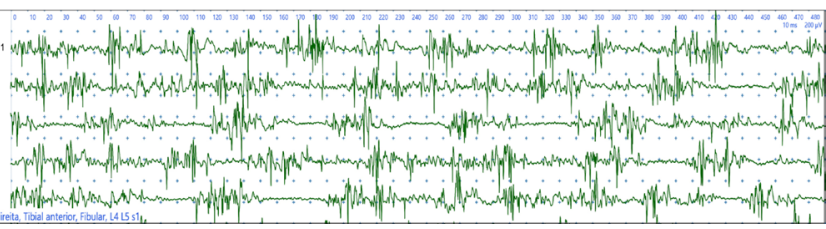

Figure 3. Myopathic potentials; tibial anterior muscle - right side.

erythematosus, with psychosis as clinical criteria and low C3 complement levels and positive anti-Smith antibody as immunological criteria, according to the 2019 American College of Rheumatology Classification Criteria for Systemic Lupus Erythematosus. Therefore, she was treated with IVIg, followed by cyclophosphamide; significant improvement was observed, and the patient was extubated. During the 2-month follow-up, only mild weakness was noted.

Neuromuscular junction and myopathic disorders are difficult to diagnose owing to their nonspecific symptoms and the necessity of specific exams to confirm the diagnosis ${ }^{2}$. During the pandemic of a respiratory virus, NMD patients are more vulnerable to infection due to thoracic musculature weakness, difficulty in mobilizing secretions, and the use of immunosuppressive medications ${ }^{3}$. SARS-CoV-2 infection can lead to neuromuscular manifestations, such as myasthenia gravis ${ }^{4,5}$, and previous myasthenia gravis can worsen the course of COVID-19 infection ${ }^{6}$.

A recent national paper described the clinical course of hospitalized patients with previous myasthenia gravis infected by COVID-19, highlighting elevated rates of mechanical ventilation and multiple antibiotics used, such as the case described ${ }^{6}$. Moreover, SARS-CoV-2 can exacerbate previous NMD that were not diagnosed due to misdiagnosis as fatigue or other psychiatric disorders, as reported in these cases $^{4}$. We aimed to emphasize the importance of investigating NMD diagnosis during a pandemic as early detection and treatment can provide patients a better prognosis.

\section{References}

1. Studart-Neto A, Guedes BF, Tuma RLE, Camelo Filho AE, Kubota GT, lepsen BG, et al. Neurological consultations and diagnoses in a large, dedicated COVID-19 university hospital. Arq Neuro-Psiquiatr. 2020 Aug;78(8):494-500. https://doi.org/10.1590/0004-282×20200089

2. Lindstrom $\mathrm{H}$, Ashworth NL. The usefulness of electrodiagnostic studies in the diagnosis and management of neuromuscular disorders. Muscle Nerve. 2018 Aug;58(2):191-6. https://doi.org/10.1002/mus.26126

3. Angelini C, Siciliano G. Neuromuscular diseases and Covid-19: Advices from scientific societies and early observations in Italy. Eur J Transl Myol. 2020 Jun;30(2):286-90. https://doi.org/10.4081/ ejtm.2019.9032
4. Paliwal VK, Garg RK, Gupta A, Tejan N. Neuromuscular presentations in patients with COVID-19. Neurol Sci. 2020 Nov;41(11):3039-56. https://doi.org/10.1007/s10072-020-04708-8

5. Sriwastava S, Tandon M, Kataria S, Daimee M, Sultan S. New onset of ocular myasthenia gravis in a patient with COVID-19: a novel case report and literature review. J Neurol. 2020 Oct;1-7. https://doi. org/10.1007/s00415-020-10263-1

6. Camelo-Filho AE, Silva AMS, Estephan EP, Zambon AA, Mendonça $\mathrm{RH}$, Souza PV, et al. Myasthenia gravis and COVID-19: clinical characteristics and outcomes. Front Neurol. 2020 Sep;11:1053. https://doi.org/10.3389/fneur.2020.01053 